\title{
Development and validation of an immunosensor for monocyte chemotactic protein 1 using a silicon photonic microring resonator biosensing platform
}

\author{
Enrique Valera, Winnie W. Shia, and Ryan C. Bailey* \\ Department of Chemistry, University of Illinois at Urbana - Champaign, \\ 600 South Matthews Avenue, Urbana, IL 61801, United States
}

*corresponding author (baileyrc@illinois.edu) 


\section{Abstract}

a) Objectives:

We report the development of an optical immunosensor for the detection of monocyte chemotactic protein 1 (MCP-1) in serum samples. MCP-1 is a cytokine that is an emerging biomarker for several diseases/disorders, including ischemic cardiomyopathy, fibromyalgia, and some cancers.

\section{b) Design and Methods:}

The detection of MCP-1 was achieved by performing a sandwich immunoassay on a silicon photonic microring resonator sensor platform. The resonance wavelengths supported by microring sensors are responsive to local changes in the environment accompanying biomarker binding. This technology offers a modularly multiplexable approach to detecting analyte localization in an antibody-antigen complex at the sensor surface.

c) Results:

The immunosensor allowed the rapid detection of MCP-1 in buffer and spiked human serum samples. An almost 2 order of magnitude linear range was observed, between 84.3 and $1582.1 \mathrm{pg} / \mathrm{mL}$ and the limits of blank and detection were determined to be 0.3 and $0.5 \mathrm{pg} / \mathrm{mL}$, respectively. The platform's ability to analyze MCP-1 concentrations across a clinically-relevant concentration range was demonstrated.

d) Conclusions: 
A silicon photonic immunosensor technology was applied to the detection of clinicallyrelevant concentrations of MCP-1. The performance of the sensor was validated through a broad dynamic range and across a number of suggested clinical cut-off values. Importantly, the intrinsic scalability and rapidity of the technology makes it readily amenable to the simultaneous detection of multiplexed biomarker panels, which is particularly needed for the clinical realization of inflammatory diagnostics.

Keywords: Cytokine; Diagnostic; Immunoassay; Optical biosensor; Silicon photonics 


\section{Introduction}

Major advances in the field of biomolecular detection have recently led to improvements in the sensitivity and specificity achievable for the detection of a myriad of biomarker targets. Many of these technologies have been applied to clinical diagnostics, where the analysis of biomarker signatures can be utilized to identify and monitor a wide spectrum of human diseases and disorders [1-5]. Due to their important roles in modulating the immune response and inflammation, cytokines are attracting increasing attention for clinical diagnostics [6-8]. Cytokines, a broadly defined category of small $(\sim 5-20 \mathrm{kDa})$ proteins that include chemokines, interleukins, interferons, lymphokines, and tumor necrosis factors, regulate many types of cellular interactions in response to both self- and non-self antigens. Not surprisingly, alterations of cytokine levels can be diagnostic for a wide variety of maladies, including autoimmune disorders, cancer, and pathogenic infections. Importantly, the low basal concentrations and overlapping functions of cytokines, coupled with the acute nature of many inflammatory conditions conspire to demand high analytical specifications for cytokine-based clinical diagnostics. To fully realize the promise of cytokine-based diagnostics, new technologies that deliver robust and cost effective performance with high sensitivity $(\mathrm{pg} / \mathrm{mL})$, specificity, and rapid time-to-result are needed.

Of particular relevance to this manuscript is monocyte chemotactic protein 1 (MCP1, also known as CCL2). MCP-1 is a low molecular weight ( 13 kDa), 76-amino acid protein, that belongs to the CC chemokine family. MCP-1 is implicated in pathogeneses of several diseases such as ischemic cardiomyopathy [9], fibromyalgia syndrome [10], or systemic lupus erythematosus [11]. It is also related to the rare neurological disorder 
Miller Fisher syndrome [12], and proposed as a biomarker for ovarian cancer [13]. The concentrations of clinical interest of this cytokine vary considerably depending on its clinical application; however, studies have defined MCP-1 cut-off values of $130 \mathrm{pg} / \mathrm{mL}$ for fibromyalgia syndrome [10], $187 \mathrm{pg} / \mathrm{mL}$ in neonates with hypoxic-ischemic encephalopathy [14], and $718 \mathrm{pg} / \mathrm{mL}$ for prognosing ovarian cancer [13].

As an alternative to plate- and bead-based immunoassays, our group has investigated a silicon photonic microring resonator technology that leverages robust and cost effective semiconductor fabrication techniques to create a sensitive and modularly multiplexable biomolecular detection platform. Microring resonators are chip-integrated optical microcavities that support the propagation of optical modes that are extremely sensitive to the local refractive index environment. Specifically, photons of a particular wavelength will only propagate in the microring under a tightly held resonance condition.

$$
m \lambda=2 \pi r n_{e f f}
$$

In Equation 1, $m$ is a non-zero integer, $\lambda$ is the wavelength of light, $r$ is the radius of the resonator, and $n_{\text {eff }}$ is the effective refractive index sampled by the optical mode. Importantly, biomolecular binding events at the microring surface lead to a local change in refractive index, which in turn leads to a shift in the resonance wavelengths supported by the device. The shifts in particular resonance wavelengths can then be tracked for individual sensors and utilized to quantitate unknown amounts of biomolecular targets. Our group has previously demonstrated the applicability of this technology to detect a several different classes of biologically-relevant targets, including proteins, nucleic acids, viruses, and biotoxins [15-18]. We have also demonstrated several different 
signal enhancement strategies on the silicon photonic platform [19-21] that deliver limits of detection comparable with many commercial immunoassays.

In this manuscript we describe the development of a high-performing silicon photonic immunosensor for MCP-1. Using an enzymatically-enhanced, sandwich immunoassay, we were able to sensitively detect this representative cytokine at sub$\mathrm{pg} / \mathrm{mL}$ levels with a relatively rapid $(71 \mathrm{~min})$ time-to-result. We demonstrate the ability to quantitate MCP-1 almost 2 orders of magnitude linear range in both buffer and human serum samples. We find minimal matrix effects when detecting in serum with full signal recovery for samples within the assay working range achieved by a simple 10-fold dilution of the sample. Importantly, we show the ability to clearly detect MCP-1 concentrations at the previously defined, clinically-relevant cut-off values for the biomarker. The biologically-relevant performance metrics of this technology, coupled with the capability to perform multiplexed detection, position this technology as an attractive platform for inflammatory cytokine-based clinical diagnostics.

\section{Materials and Methods}

\subsection{Instrumentation}

Resonance wavelength shifts were monitored using the Maverick Detection System (Genalyte, Inc., San Diego, CA). The pH of all buffers and solutions were measured with an Orion 3-star benchtop pH meter (Thermo Scientific). Data analysis was performed using OriginPro 9.1.0 (OriginLab Corporation, Northampton, MA) and calibration curves were fit with a four-parameter logistic equation using GraphPad Prism 5 for Windows 
(GraphPad Software, San Diego, CA). Data presented corresponds to the average of at least 16 on-chip technical replicates per concentration of MCP-1.

\subsection{Chemical and biochemical reagents}

Dulbecco's phosphate buffered saline packets were purchased from Sigma-Aldrich (St. Louis, MO). 3-aminopropyltriethoxysilane (APTES) (cat. num. 80370), bis[sulfosuccinimidyl] suberate (BS3, cat. num. 21585), streptavidin-HRP conjugate (cat. num. 21130), 1-step 4-chloro-1-naphthol (4-CN) solution, and StartingBlock (PBS) blocking buffer (cat. num. 37538) were purchased from Thermo Scientific. DryCoat assay stabilization reagent was purchased from Virusys (cat. num. AG066-1) and glycerol (cat. num. BP229-1) from Fisher BioReagents. The capture antibody (antiHuman MCP-1 (CCL2), cat. num. 14-7099), detection antibody (biotinylated anti-MCP-1 (CCL2), cat. num. 13-7096), and the target analyte (recombinant human protein MCP-1 (CCL2), cat. num. 14-8398) were purchased from eBioscience (San Diego, CA). The non-specific adsorption control antibody (Mouse IgG, cat. num. ab37355) was purchased from abcam (Cambridge, MA).

\subsection{Buffers and solutions}

PBS buffer (10 mM) was reconstituted from Dulbecco's phosphate buffered saline packets (D5773 Sigma) and the pH was adjusted to 7.4. The MCP-1 capture antibody was buffer exchanged to $10 \mathrm{mM}$ PBS, followed by addition of glycerol to a final $5 \%(\mathrm{v} / \mathrm{v})$ glycerol in PBS. The assay running buffer was $0.5 \%$ BSA in $10 \mathrm{mM}$ PBS. All buffer solutions were prepared with purified water (ELGA PURELAB filtration system; Lane End, UK). 


\subsection{Human serum samples}

Certified pooled human serum from healthy individuals was obtained from Innovative research (Novi, MI). The test material was aliquotted upon receipt and stored at $-20^{\circ} \mathrm{C}$ until to use.

\subsection{Silicon photonic microring resonators: Sensor substrates and read-out} instrumentation

The Maverick M1 optical scanning instrumentation utilized to measure shifts in microring resonance wavelengths and sensor substrates were obtained from Genalyte, Inc. The fabrication of the sensor chip and scanning instrumentation operation used has been described previously $[22,23]$. The $4 \mathrm{~mm} \times 6 \mathrm{~mm}$ silicon-on-insulator chips each contain 128 individually-addressable microrings. Four additional microrings in the fluidic channel, but covered by a fluoropolymer cladding layer, serve as controls to correct for thermal drift. An additional 4 exposed microrings (no cladding layer) lie outside the fluidic channel and serve as leak sensors.

Each individual microring is located next to an adjacent linear waveguide, such that interference between photons circulating the microring and passing down the linear waveguide create a resonant microcavity that supports optical modes only at specific wavelengths [23]. The configuration of the fabricated chip allows for division of the 128 microrings in two fluidically-addressable flow channels, as defined by a laser cut Mylar gasket that is sandwiched between the chip and Teflon lid. This fluidic design allows two unique samples to be assayed simultaneously. 
To measure the resonance wavelength shift associated with the steps of the immunoassay, a tunable external cavity diode laser centered at $1550 \mathrm{~nm}$ serially probes each microring individually as a function of time. Resonances were determined as dips in the intensity of light propagating down the linear waveguide past the microring as the laser wavelength is scanned across a suitable spectral window. Relative shifts in resonance wavelength, presented in units of picometers $(\mathrm{pm})$, were recorded as a function of time during each of the immunoassay steps. The data acquisition software enables real-time control subtraction and averaging of active sensor responses.

\subsection{Surface functionalization}

Before functionalization, sensor chips were briefly rinsed with acetone to remove a protective photoresist coating. Sensor chips were immersed into a $5 \%(\mathrm{v} / \mathrm{v})$ solution of APTES ( $2 \mathrm{~mL}$ in acetone, $4 \mathrm{~min}$, with stirring) and then sequentially rinsed in acetone (2 min), isopropanol (2 min), and water. Silanized chips were gently dried under a $\mathrm{N}_{2}$ stream, reacted with a $5 \mathrm{mM}$ solution of the BS3 crosslinker $(20 \mu \mathrm{L}, 2 \mathrm{mg}$ in $700 \mu \mathrm{L} 2$ $\mathrm{mM}$ acetic acid) for $3 \mathrm{~min}$, and again dried with $\mathrm{N}_{2}$.

Solutions of capture and control antibodies $(0.3 \mathrm{mg} / \mathrm{mL}$, in $10 \mathrm{mM}$ PBS with $5 \%$ glycerol) were immobilized onto specific regions in both channels of the chip via microspotting (0.2 $\mu \mathrm{L}$ per drop). Antibody solutions were reacted for $1 \mathrm{~h}$ at RT. The chips were then immersed in the blocking solution (600 $\mu \mathrm{L}$, StartingBlock) for $1 \mathrm{~h}$ at $\mathrm{RT}$. After blocking, the chips were dip-coated with DryCoat (600 $\mu \mathrm{L} ; 30$ dips). After this procedure, the chips were stored in a desiccator at $4^{\circ} \mathrm{C}$ until use. 


\subsection{Immunosensor measurement procedure}

Before each measurement, the chips were loaded into a base cartridge holder and then sandwiched between a 0.007 " laser cut Mylar gasket, and a Teflon cartridge top. Solutions were delivered to the cartridge assembly via a 0.01 " ID Teflon tubing screwed directly into the cartridge top. The reagents in the assay were diluted in degassed running buffer, and loaded into a 96-well plate.

The Maverick system control software enables fully programmable reagent delivery. For all steps in the assay, the flow rate was $30 \mu \mathrm{L} / \mathrm{min}$. Before starting the scan, the flow across the system was checked by a pre-buffer rinse step ( $<4$ min, running buffer). Solutions were flowed across both channels of the chip in the following order: 1) running buffer (2 min); 2) sample (20 min); 3) running buffer (2 min); 4) biotinylated detection antibody (10 min, $4 \mu \mathrm{g} / \mathrm{mL}$ ); 5) running buffer (2 min); 6) streptavidin-HRP conjugate (10 min, $4 \mu \mathrm{g} / \mathrm{mL}$ ); 7) running buffer (3 min); 8) 4-CN (15 min, $4 \mu \mathrm{g} / \mathrm{mL}$ ); 9) running buffer (7 $\min )$. The total assay time was 71 minutes.

\subsection{Data analysis and processing}

The resonance wavelength shifts, related to the MCP-1 concentration, were calculated using the OriginPro 9.1.0 software, after subtracting responses from thermal and leak rings. Standard curves were constructed using GraphPad Prism 5 for Windows by plotting the resonance shift as a function of MCP-1 concentration and fitting to a logistic four-parameter equation:

$$
Y=(A-B) /\left[1+(x / C)^{D}\right]+B
$$


where $A$ is the shift measured for the highest concentration of target, $B$ is the shift measured for the lowest concentration of target, $C$ is the concentration producing $50 \%$ of the maximum response, and $D$ is the slope at the inflection point of the sigmoid curve. The working range was determined to be in the interval between $20 \%$ and $80 \%$ of the $A$ value.

The limit of blank (LoB) and limit of detection (LoD) were calculated according to a standard approach [24].The LoB was calculated to be the concentration equaling the average response from microrings in a negative control blank experiment (no MCP-1) plus 3 times the standard deviation from this blank measurement $(n=16)$. The LoD is defined as the lowest concentration likely to be measured above the LoB, and is calculated according to:

$$
L O D=L O B+\left(c_{\beta} \times S D_{S}\right)
$$

where $S D_{S}$ is the analytical standard deviation of a sample with the lowest measured concentration $(0.2 \mathrm{pg} / \mathrm{mL})$; and $c_{\beta}$ is approximately 1.65 [24].

\section{Results}

The objective of this work was to validate the ability of the silicon photonic microring resonator platform to measure MCP-1 in buffer and human serum samples, and to establish quantitative detection metrics. The sensor array was first created by covalently immobilizing capture and control antibodies onto discrete sensor elements (Figure 1a). Chips were assembled into a fluidic cartridge (Figure 1b) and loaded into the Maverick detection system for analysis. 
A pair of commercial antibodies against MCP-1 (capture and detection) was identified and utilized in a sandwich immunoassay, each step of which was monitored in real time using the silicon photonic detection platform (Figure 1c). After functionalization and establishing baseline in running buffer $(t=0 \mathrm{~min}$, and between subsequent reagent steps throughout assay), standard solutions of MCP-1 (between 0 and $50000 \mathrm{pg} / \mathrm{mL}$, prepared in running buffer) were flowed across the chip surface ( $\mathrm{t}=2 \mathrm{~min})$. Biotinylated detection antibodies were then introduced $(\mathrm{t}=24 \mathrm{~min})$, followed by a streptavidin-HRP conjugate $(t=36 \mathrm{~min})$. The $4-\mathrm{CN}$ solution was then introduced $(\mathrm{t}=49 \mathrm{~min})$ and flowed over the chip for $15 \mathrm{~min}$, and the catalytic conversion of $4-\mathrm{CN}$ to the insoluble $4-\mathrm{CNP}$ was measured by subtracting the net resonance wavelength shift from running buffer steps immediately before and after the 4-CN/4-CNP step. The magnitude of resonance wavelength shift from the $4-C N P$ step (calculated from $t=49 \min$ to $t=70 \mathrm{~min}$ ) was related to the concentration of MCP-1 concentration in each standard solution.

The shift in resonance wavelength observed for different concentrations of MCP-1 is shown in Figure 2a. This same data is plotted as a dose-response curve in Figure $2 b$ (for detailed values see Table 1), and from this data the LoB and LoD were determined to be 0.3 and $0.5 \mathrm{pg} / \mathrm{mL}$, respectively, and the linear range of the assay was between 84.3 and $1582.1 \mathrm{pg} / \mathrm{mL}$. Responses for microrings functionalized with a control antibody were negligible.

Turning to the analysis of human serum samples, the effects of the sample matrix on sensor response was determined. To accomplish this, pooled human serum samples were spiked with a high concentration of MCP-1 $(10,000 \mathrm{pg} / \mathrm{mL})$. At this concentration the response is very near the saturated region of the calibration curve, meaning that any 
small amount of MCP-1 natively present in the human sample would not significantly change the sensor response. This allows for the direct observation of matrix effects. Figure $2 \mathrm{c}$ shows the observed resonance wavelength shifts recorded under different dilutions of the spiked serum sample, when compared to equivalent concentrations measured in running buffer, after correction for dilution. A 1:1 dilution showed a $\sim 15 \%$ reduction in response. A 1:5 dilution reduced this matrix effect and a 1:10 dilution showed full response recovery for samples measured within the assay working range. Further dilution (1:20) did not improve the response recovery.

Working at this optimized 1:10 dilution, we analyzed human serum samples spiked with concentrations of MCP-1 surrounding clinically-established cut-off values associated with specific diseases/disorders. Specifically, cut-offs at 130 and $710 \mathrm{pg} / \mathrm{mL}$ have been established for fibromyalgia syndrome [10] and ovarian cancer [13], respectively. After 1:10 dilution, serum samples were spiked with 13, 30, 71.8 and 100 $\mathrm{pg} / \mathrm{mL}$ of MCP-1. A non-spiked serum sample was used as reference. The measured resonance wavelength shifts for each of the spiked samples ( $n \geq 23$ microring sensors for each sample) were interpolated to the buffer calibration curve, and the resulting MCP-1 concentrations determined are plotted in Figure 3. The concentration calculated from the non-spiked serum sample was used to correct the other concentrations by subtracting this baseline concentration. The good agreement between the spiked values and those determined using the microring resonator technology is numerically illustrated in Table 2. Both of these representations illustrate that the microring immunosensor is capable of quantitating concentrations of $\mathrm{MCP}-1$ in human serum samples. This 
analysis also allowed determination of the concentration of MCP-1 natively present in the serum sample to be $56 \pm 16 \mathrm{pg} / \mathrm{mL}$.

\section{Discussion}

Silicon photonic microring resonators are an emerging optical sensing technology that is attractive for biomolecular diagnostic applications. This refractive-index sensitive technology can be operated in a label-free mode wherein the biomolecular target of interest does not have to be covalently modified with any chromogenic, fluorescent, or enzymatic tag. However, the use of multiple, high affinity, target-specific recognition elements (i.e. monoclonal antibodies) allows this technology to be deployed for the quantification of disease-relevant biomarkers within clinically-relevant matrices. In this manuscript, we utilize arrays of silicon photonic microring resonators in a sandwich immunoassay format for the detection of MCP-1, an inflammatory cytokine associated with a number of clinically-relevant diseases/disorders, validate the assay in serum samples, and demonstrate many important analytical parameters.

The natively passivated silicon oxide present on the resonators makes it amenable to standard silane chemistries and bioconjugate techniques, analogous to those used in many conventional microrarrays. The sensors were first covalently modified with a monoclonal antibody specific for MCP-1. Importantly, the small size of the sensor minimizes reagent consumption such that $<1 \mu \mathrm{L}(0.3 \mathrm{mg} / \mathrm{mL})$ was needed to achieve a large number ( $n \geq 23$ ) of technical replicates on a single chip. After functionalization, MCP-1 standards or human serum samples were flowed across the antibodyfunctionalized sensor array. To ensure assay specificity within complex matrices, a 
biotinylated, monoclonal anti-MCP-1 monoclonal antibody targeting a different epitope was introduced as a tracer antibody. Though the resonance wavelength shift accompanying the binding of the tracer antibody is observable above the noise baseline at many concentrations, the signal can be greatly increased through an enzymatic enhancement step. Specifically, a streptavidin-HRP conjugate that recognizes the surface-localized, biotinylated tracer antibody is introduced. This enzyme can then catalytically convert a soluble $4-\mathrm{CN}$ reagent into an insoluble 4-CNP product that is physically deposited on the microring surface [21].

For the detection of MCP-1 on the silicon photonic microring resonator platform, the entire sandwich immunoassay took a total of 71 minutes and each step was monitored in real-time, as shown in Figures $1 \mathrm{c}$ and $2 \mathrm{a}$. By comparison, commercially available ELISA kits do not allow real-time visualization of each step and typically require much longer assays (R\&D systems, 3.5 or $4.5 \mathrm{~h}$ [25]; Thermo Scientific, 3h [26]). The ability to watch each assay step in real time permits the observation of gross failures that might otherwise not be revealed until the final stage of assay read-out. The resonance wavelength shift from this final enzymatic enhancement step is measured and utilized to generate a standard curve and quantitate unknown levels of MCP-1 (Figure 2b). Statistical analysis of the standard curve yielded LoD and LoB values of 0.5 and 0.3 $\mathrm{pg} / \mathrm{mL}$, respectively, and highlighted a broad 2-order of magnitude dynamic range up to $\sim 1600 \mathrm{pg} / \mathrm{mL}$ (Table 1). Across this dynamic range, CVs were generally $\leq 10 \%$. Importantly, these values compare well with both plate- and bead-based commercial assays for MCP-1,[25-27] which report sensitivities ranging from $0.47-10 \mathrm{pg} / \mathrm{mL}$, similar working ranges, and CVs. Importantly, by requiring just over $1 \mathrm{~h}$ the microring 
immunosensor is considerably more rapid than these commercial immunoassays. Additionally, while not the focus of this report, antibodies specific for multiple targets can be simultaneously arrayed onto different microring sensor elements on a single chip allowing for high levels of multiplexing, similar to Luminex.[27] Therefore, the simultaneous quantitative analysis of multiple cytokine/chemokines should be readily achievable using the microring detection technology.

Essential to any clinically-relevant assay is the ability to assess and compensate for any matrix effects. To probe these effects, we spiked a known concentration of MCP-1 into human serum samples and found that only minor signal attenuation was observed (Figure 2c) and that full response recovery could be achieved with only a 1:10 dilution of the serum samples into running buffer. Using this dilution, we then validated the ability to detect MCP-1 from within spiked serum at concentrations that corresponded to clinically-relevant cut-off values suggested for both fibromyalgia syndrome and ovarian cancer. The silicon photonic immunosensor was clearly able to distinguish baseline serum levels from these cut-off values with the measured shifts in resonance wavelength being quantitative through extrapolation to the standard calibration curve and with good response recovery through the linear working range (Table 2). Values for the lower spiked concentrations showed a reduced percent recovery, mainly because these concentrations are out of the working range of the calibration curve; however, the absolute values are within $15 \mathrm{pg} / \mathrm{mL}$ of the spiked concentration.

Beyond the scope of this manuscript, it is worthwhile to mention that the current generation sensor chip has the capacity to be functionalized with up to 32 target-specific capture antibodies, which in principle would allow for high levels of multiplexing. 
Coupled with the rapidity and biologically-relevant analytical performance metrics, we feel that this silicon photonic detection platform could be a valuable tool for translational applications of multiplexed cytokine and other biomarker-based diagnostics.

\section{Conclusions}

A silicon photonic immunosensor technology was applied to the detection of clinically-relevant concentrations of MCP-1. The performance of the sensor was validated through a broad dynamic range and across a number of suggested clinical cut-off values. Performance metrics were comparable to commercial ELISA assays for this biomarker; however, the silicon photonic immunoassay was considerably more rapid. Importantly, the intrinsic scalability of the technology makes it readily amenable to the simultaneous detection of multiplexed biomarker panels, which is particularly needed for the clinical realization of inflammatory marker-based diagnostics.

\section{Acknowledgements}

We gratefully acknowledge financial support from the National Science Foundation (CHE 12-14081) and National Cancer Institute (R33-CA177462-01). WWS was supported by an American Heart Association Predoctoral Fellowship 


\section{Figure Captions}

Fig. 1. a) Schematic representation of the microring arrays on the sensor chip and the distribution of immobilized antibodies on chip. The antibodies were spotted in an identical formal over the two channels; b) Image of a chip assembled inside of the fluidic cartridge used; c) Overall strategy for the HRP-enhanced detection of MCP-1. MCP-1 concentration $=50000 \mathrm{pg} / \mathrm{mL}$ (buffer).

Fig. 2. a) Data recorded with the developed immunosensor. MCP-1 standard solutions prepared in running buffer; b) Representative calibration curve (black curve, running buffer). See Table 1 for details. Each data point shown corresponds with the average of at least 23 replicates. The red curve corresponds to the signal of the control antibody; c) Matrix effect of pooled human serum.

Fig. 3. Concentrations around MCP-1 cut-offs values, 10-fold, were spiked (13, 30, 71.8 and $100 \mathrm{pg} / \mathrm{mL}$ ) to the 10 times diluted serum samples. The signal obtained was converted to a MCP-1 concentration via the buffer calibration curve. Inset: correlation between the spiked samples and the measured values by the developed immunosensor. The non-spike serum sample was used as reference.

\section{Table 1}

Detailed values of the MCP-1 immunosensor calibration curve in running buffer

\section{Table 2}

Analysis of spiked human serum samples using the MCP-1 immunosensor 
Valera et al.

Table 1

\begin{tabular}{|l|c|}
\hline & MCP-1 \\
Immunosensor * & $53.9 \mathrm{pm}$ \\
Signal $_{\min }$ & $7833 \mathrm{pm}$ \\
Signal & \\
Slope & 0.79 \\
LoB & $0.3 \mathrm{pg} / \mathrm{mL}$ \\
LoD & $0.5 \mathrm{pg} / \mathrm{mL}$ \\
Working range & $84.3-1582.1 \mathrm{pg} / \mathrm{mL}$ \\
$\mathbf{R}^{2}$ & 0.998 \\
\hline
\end{tabular}

* $\mathrm{S} / \mathrm{N}=145$. The standards were measured with the immunosensor following the procedure described in the experimental section. The parameters were extracted from the four-parameter equation used to fit the standard curve. 


\begin{tabular}{|l|l|l|l|}
\hline Human Serum Samples (1/10 dilution) * & \multicolumn{2}{l|}{} \\
\hline $\begin{array}{l}\text { Spiked concentrations } \\
(\mathbf{p g} / \mathrm{mL})\end{array}$ & $\begin{array}{l}\text { Measured concentrations } \\
(\mathbf{p g} / \mathrm{mL})\end{array}$ & $\begin{array}{l}\text { Recovery } \\
(\%)\end{array}$ & $\begin{array}{l}\text { RSD } \\
(\%)\end{array}$ \\
\hline $\mathbf{1 3}$ & $5.31 \pm 3.66$ & 40.8 & 28.2 \\
$\mathbf{3 0}$ & $15.58 \pm 4.83$ & 51.9 & 16.1 \\
$\mathbf{7 1 . 8}$ & $67.57 \pm 6.95$ & 94.1 & 9.7 \\
$\mathbf{1 0 0}$ & $94.90 \pm 17.18$ & 94.9 & 17.2 \\
\hline
\end{tabular}

* The non-spike serum sample was used as reference.

The parameters were extracted from the four-parameter equation used to fit the standard curve. The measured concentrations were obtained after subtract the native MCP-1 concentration in the serum samples $(5.6 \mathrm{pg} / \mathrm{mL}, 1 / 10$ dilution). 


\section{References}

1. Liotta L, Petricoin E. Molecular profiling of human cancer. Nature Reviews Genetics. 2000;1:48-56.

2. Zhang $X$, Li L, Wei D, Yap Y, Chen F. Moving cancer diagnostics from bench to bedside. Trends Biotechnol. 2007;25:166-73.

3. Tothill IE. Biosensors for cancer markers diagnosis. Semin Cell Dev Biol. 2009;20:5562.

4. Teles FSRR. Biosensors and rapid diagnostic tests on the frontier between analytical and clinical chemistry for biomolecular diagnosis of dengue disease: A review. Anal Chim Acta. 2011;687:28-42.

5. McNerney R, Daley P. Towards a point-of-care test for active tuberculosis: Obstacles and opportunities. Nature Reviews Microbiology. 2011;9:204-13.

6. Kajikawa O, Goodman RB, II MCJ, Kiyoshi K, Martin TR. Sensitive and specific immunoassays to detect rabbit il-8 and mcp-1: Cytokines that mediate leukocyte recruitment to the lungs. J Immunol Methods. 1996;197:19-29.

7. Freer G, Rindi L. Intracellular cytokine detection by fluorescence-activated flow cytometry: Basic principles and recent advances. Methods. 2013;61:30-8.

8. Jager Wd, Velthuis Ht, Prakken BJ, Kuis W, Rijkers GT. Simultaneous detection of 15 human cytokines in a single sample of stimulated peripheral blood mononuclear cells. Clinical and Diagnostoc Laboratory Immunology. 2003;10:133-9.

9. Frangogiannis NG, Dewald O, Xia Y, Ren G, Haudek S, Leucker T, et al. Critical role of monocyte chemoattractant protein-1/cc chemokine ligand 2 in the pathogenesis of ischemic cardiomyopathy. Circulation. 2007;115:584-92.

10. Blanco I, Janciauskiene S, Nita I, Fernández-Bustillo E, Cárcaba V, Gallo C, et al. Low plasma levels of monocyte chemoattractant protein-1 (mcp-1), tumor necrosis factor-alpha (tnfa), and vascular endothelial growth factor (vegf) in patients with alpha1antitrypsin deficiency-related fibromyalgia. Clin Rheumatol. 2010;29:189-97.

11. Barbado J, Martin D, Vega L, Almansa R, Gonçalves L, Nocito M, et al. Mcp-1 in urine as biomarker of disease activity in systemic lupus erythematosus. Cytokine. 2012;60:583-6.

12. Sato S, Suzuki K, Nagao R, Kashiwagi Y, Kawashima H, Tsuyuki K, et al. Detection of mcp-1 and il-8 in the serum and cerebrospinal fluid of a child with miller fisher syndrome. Journal of Clinical Neuroscience. 2009;16:1698-9.

13. Hefler L, Tempfer C, Heinze G, Mayerhofer K, Breitenecker G, Leodolter S, et al. Monocyte chemoattractant protein-1 serum levels in ovarian cancer patients. $\mathrm{Br} \mathrm{J}$ Cancer. 1999;81:855-9.

14. Jenkins DD, Rollins LG, Perkel JK, Wagner CL, Katikaneni LP, Bass WT, et al. Serum cytokines in a clinical trial of hypothermia for neonatal hypoxic-ischemi $\mathrm{C}$ encephalopathy. J Cereb Blood Flow Metab. 2012;32:1888-96. 
15. Washburn AL, Luchansky MS, Bowman AL, Bailey RC. Quantitative, label-free detection of five protein biomarkers using multiplexed arrays of silicon photonic microring resonators. Analytical Chemistry. 2010;82:69-72.

16. Qavi AJ, Bailey RC. Multiplexed detection and label-free quantitation of micrornas using arrays of silicon photonic microring resonators. Angewandte Chemie International Edition. 2010;49:4608-11.

17. McClellan MS, Domier LL, Bailey RC. Label-free virus detection using silicon photonic microring resonators. Biosensors and Bioelectronics. 2012;31:388-92.

18. Shia WW, Bailey RC. Single domain antibodies for the detection of ricin using silicon photonic microring resonator arrays. Analytical Chemistry. 2013;85:805-10.

19. Luchansky MS, Bailey RC. Silicon photonic microring resonators for quantitative cytokine detection and t-cell secretion analysis. Analytical Chemistry. 2010;82:1975-81.

20. Luchansky MS, Washburn AL, McClellan MS, Bailey RC. Sensitive on-chip detection of a protein biomarker in human serum and plasma over an extended dynamic range using silicon photonic microring resonators and sub-micron beads. Lab on a Chip. 2011;11:2042-4.

21. Kindt JT, Luchansky MS, Qavi AJ, Lee S-H, Bailey RC. Subpicogram per milliliter detection of interleukins using silicon photonic microring resonators and an enzymatic signal enhancement strategy. Anal Chem. 2013;85:10653-7.

22. Iqbal M, Gleeson MA, Bradley Spaugh, Tybor F, Gunn WG, Hochberg M, et al. Label-free biosensor arrays based on silicon ring resonators and high-speed optical scanning instrumentation. IEEE J Sel Topics Quantum Electron. 2010;16:654-61.

23. Washburn AL, Gunn LC, Bailey RC. Label-free quantitation of a cancer biomarker in complex media using silicon photonic microring resonators. Analytical Chemistry. 2009;81:9499-506.

24. Linnet $\mathrm{K}$, Kondratovich $\mathrm{M}$. Partly nonparametric approach for determining the limit of detection. Clin Chem. 2004;50:732-40.

25. R\&Dsystems. Human ccl2/mcp-1 quantikine elisa kit.

26. ThermoScientific. Human mcp-1 elisa kits.

27. R\&Dsystems. Luminex performance human cytokine panel a. 

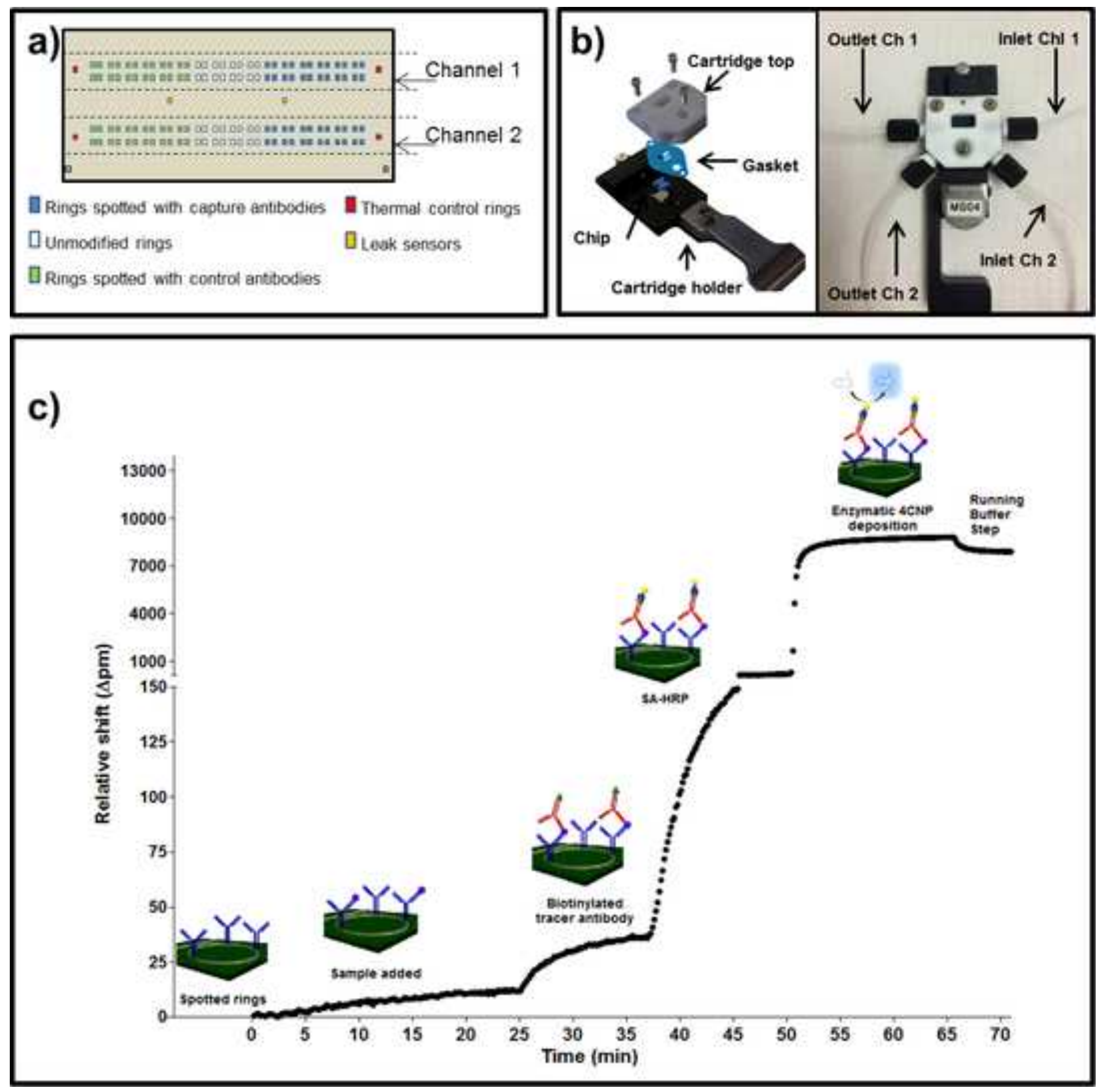
a)

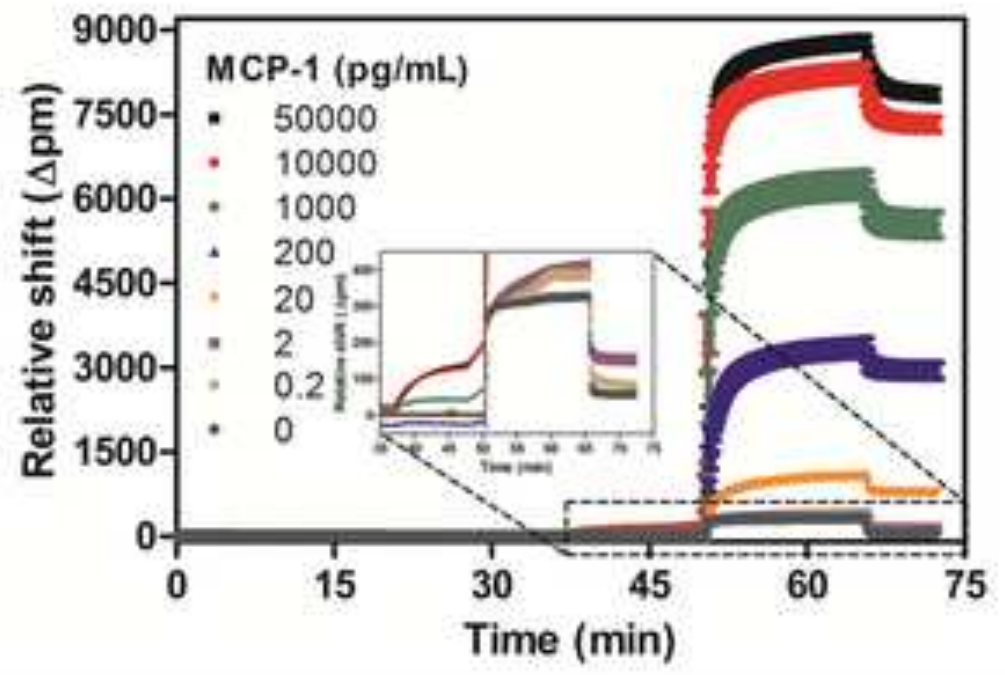

b)

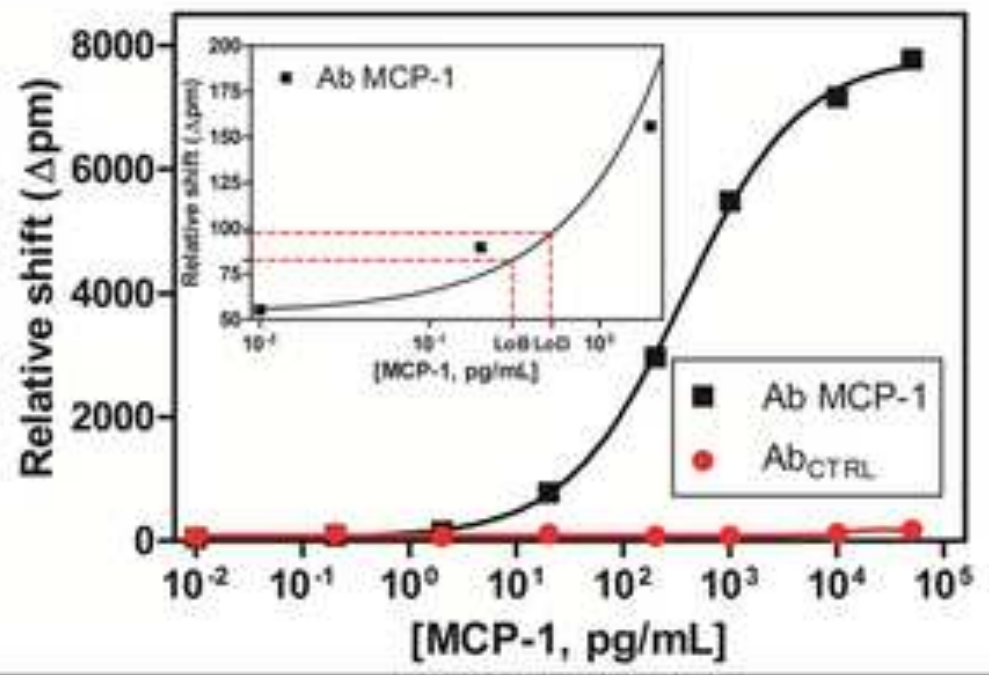

c)

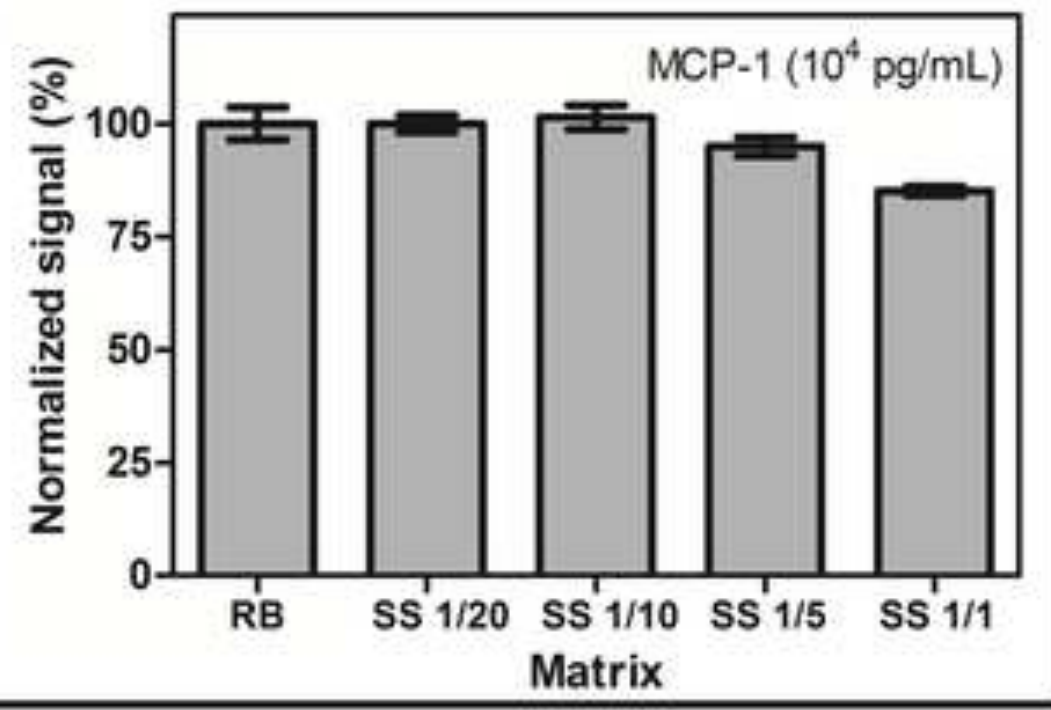




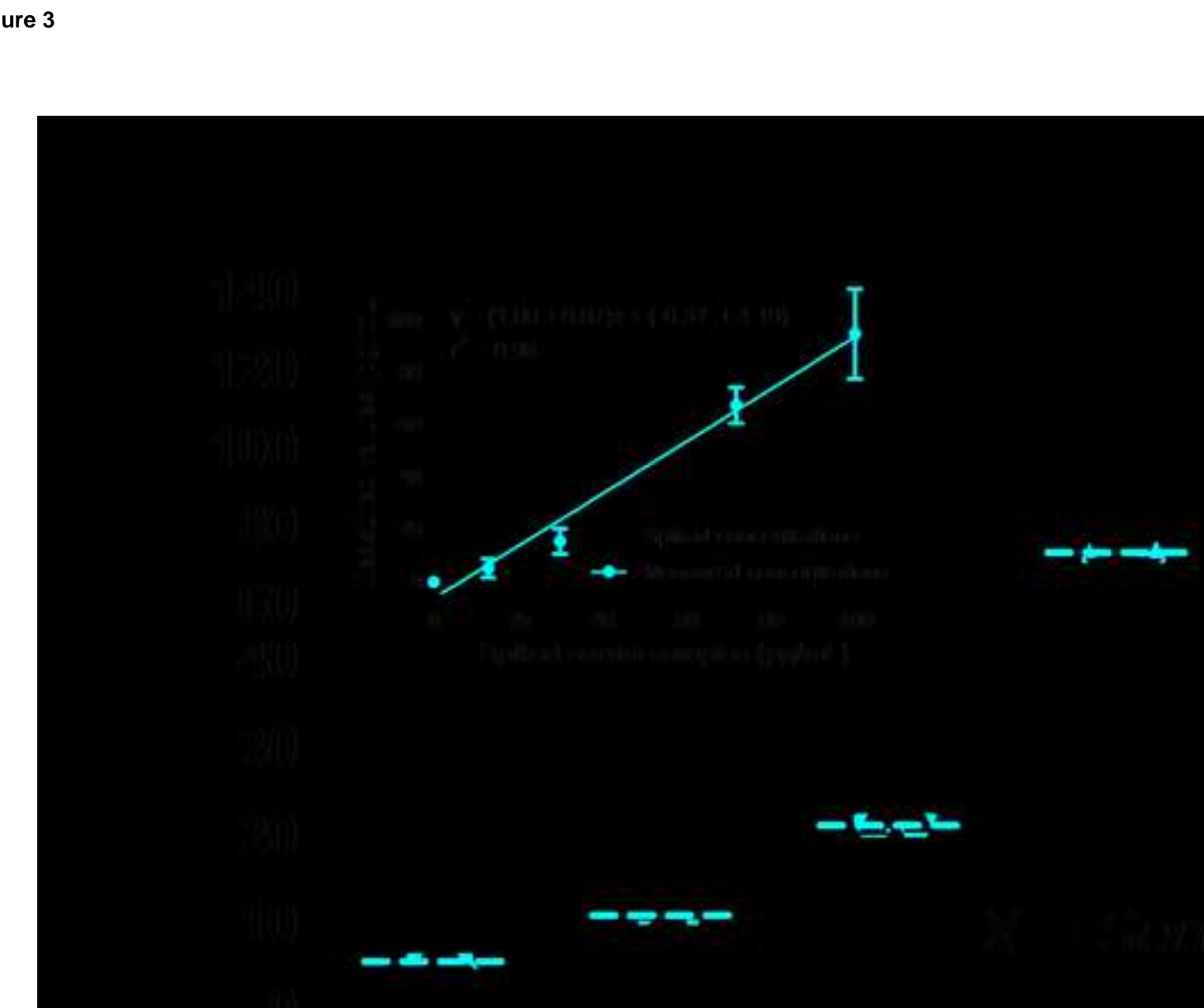

$$
\text { - th- }
$$

\section{- \\ $-\frac{1}{2}$}

$$
\begin{aligned}
& -m \\
& =-2 \\
& \text { 든 } \\
& \text { (2) }
\end{aligned}
$$

Figure 

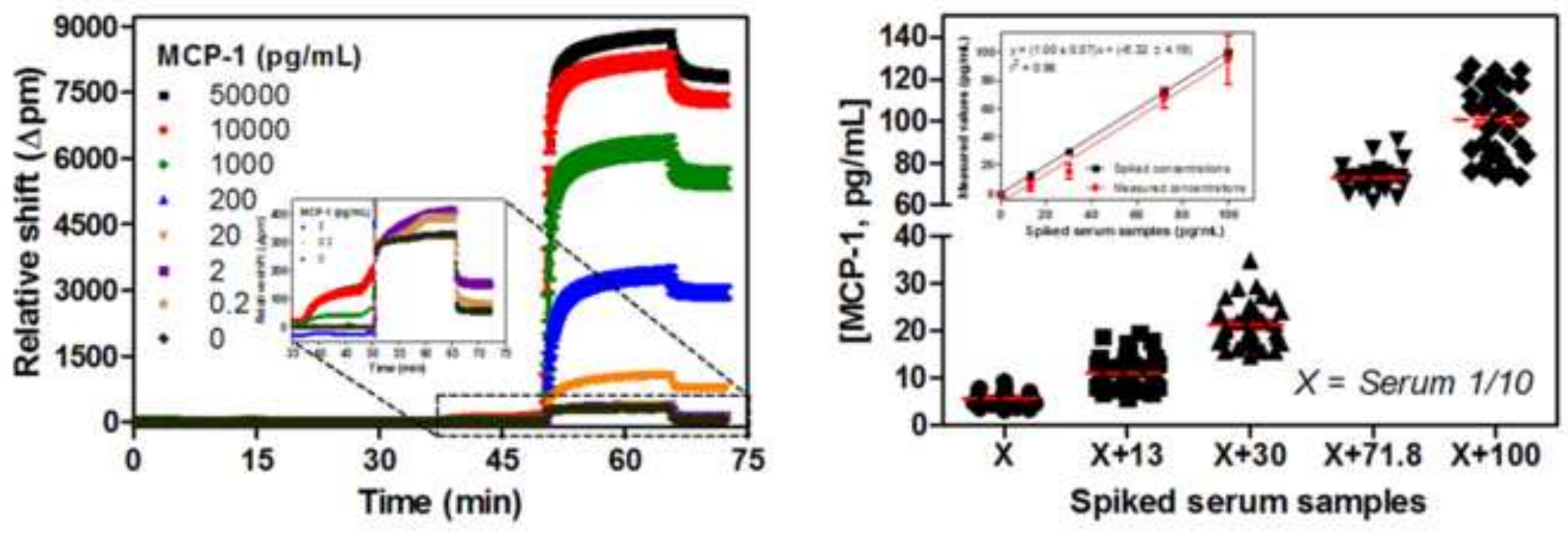